\title{
The Study and Implementation of Phong Shading Algorithm Parallelization
}

\author{
Xin Zhang \\ School of Electronic Engineering \\ Xi'an University of Posts and Telecommunications \\ Xi'an, China \\ xiaoxindexinxin@163.com
}

\author{
Jungang Han \\ ASIC Design Center \\ Xi'an University of Posts and Telecommunications \\ Xi'an, China \\ hjg@xupt.edu.cn
}

\begin{abstract}
In order to speed up operations and improve operational efficiency, The parallel algorithms of phong shading model is proposed and validated by using the simulation system for PAAG(Polymorphous Array Architecture Graphic), the compilation system, the debugging environment and the parameter extraction tool for performance analysis. The experimental results show that the parallelized implementation achieve up to 2.78 speed up.
\end{abstract}

Keywords-shading algorithm; parallelization;speedup

\section{INTRODUCTION}

In OpenGL, light and objects in the scene can be calculated, which creates many different types of threedimensional effects. As the light is indispensable in nature, drawing a realistic three-dimensional objects requires the disposing of the light. OpenGL lighting is an approximation realistic lighting. Attributes of light source in OpenGL include radiation, ambient, diffuse and specular light and so on. Material is an attribute of the object, indicating how the object is made of materials. Materials in OpenGL define the various light reflection rate of surfaces. The color ultimately reflected to the human eye by the objects in the scene is the color resulted from the multiplication of the RGB components of the light and the reflectance of the RGB components of the material. Currently used lighting algorithms in OpenGL include Gouraud model and Phong[2] model. In this paper, we focus on the parallelization[5] of Phong model algorithm.

\section{VERTEX Lighting AlgORITHMS}

\section{A. Phong Model}

Phong model is the one of the classical graphics lighting model, the model can be used to generate realistic graphics. Gouraud shading[2] using color interpolation methods to eliminate discontinuity between polygons. This can produce a smooth surface feel, each attribute of the vertex should be calculated in the stage of vertex shader, it has a relatively small amount of computation. Phong shading is similar to Gouraud shading, this method uses the color interpolation instead of vector interpolation. Results are more realistic. The work of calculation is pushed to the pixel shader stage with a relatively large amount of computation. Here is Phong lighting model algorithms.

\section{B. Phong Shading Algorithm}

Phong model supports several types of interactions between the material and light: ambient light, diffuse light and specular light, with considering the attenuation factor and the role of emitting light. For each color component, each point has several separate light sources, each component can be individually calculated. The vertex color is constituted of them. The following are separate descriptions:

- Ambient light: Ambient light is totally diffused in the environment, whose direction can't be identified. When the ambient light strikes a surface, it will be spread in all directions. The light is the ambient color according to the material properties of the environment after scaling values:

$$
\text { ambient }_{\text {light }} * \text { ambient }_{\text {material }}
$$

- Diffuse light: Diffuse light comes from a direction, the intensity of reflected light depend on not only the material but also the position of the light source.The position relative to the surface. If the light is irradiated to the front surface, it would be brighter. If the light sideways passing surface, it would be dark relatively. It is related to both the normal vector $N$ and on the unit vector $L$ between the position the light source and the vertex, just as shown in Fig.1. Diffuse light component should consider whether the light irradiated on the vertex .At the same time ,the diffuse color of the light source and properties of the diffuse material:

$$
(\max \{L \cdot n, 0\}) * \text { diffuse }_{\text {light }} * \text { diffuse }_{\text {material }}
$$

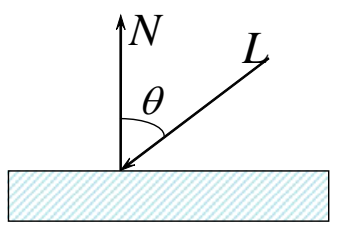

Figure 1. Reflected ray.

- Specular light : Without considering the specular light, although scene images has effect of darkness 
and lightness, as well as three-dimensional effect, it seems that there is a lackness of the highlighted area is missing on the surface. Highlighted region comes from the reflection of shiny objects. The smoother the surface, the effect is more like a mirror reflection. Its value depends on the sum of these two unit vectors: unit vector $L=\left(L_{X}, L_{Y}, L_{Z}\right)$ from the vertex to the position of the light source unit normal vector $n=\left(n_{X}, n_{Y}, n_{z}\right)$ of the vertex. At the same time, it is also related to the specular exponent shininess, specular color specular light and specular material properties specular $_{\text {material. }}$.

$\left(\max \left\{s^{*} n, 0\right\}\right)^{\text {shininess }} \times$ specular $_{\text {light }} \times$ specular $_{\text {materia }}$

- Attenuation factor: Taking into account the distance factor, the light source emitting from the light source to the surface, the light intensity gradually decreases with distance increases. Attenuation depends on the distance $d$ between the light source position and the vertex, the constant attenuation factor, linear attenuation factor, the quadratic attenuation factor .While considering whether it is the spotlight light source, we need to determine whether the light is in the spotlights and whether the vertex is in the cone of light. If it is, then we should also consider unit vector from the vertex to the spotlight, the directional of the spotlight, cut angle factor of spotlight. Attenuation factor and spotlight effects following formula:

$$
\text { attenuationfactor }=\frac{1}{k_{c}+k_{l} d+k_{q} d^{2}}
$$

$$
\text { spotlighteffects }=(\max \{v \cdot d, 0\}))^{G L_{-} S P O T_{-} E X P O N E N T}
$$

- Light emitting of material: Emission light of the material simulates the light of an object ,emission color of the surface can increase the strength of the object, its value is assigned to the RGB values of the parameter of GL_EMISSION.

- Complete lighting formula: In summary, the above results are superimposed, the vertex color[1]= material emission color + global ambient light (zoomed due to the material properties in the environment ) +ambient light components, diffuse light components and the specular lights which are properly decayed (come from all the light sources). In the implementation of lighting calculations, the color values is within the range in $[0,1]$, Light formula is showing as follows.

$$
\begin{aligned}
& \text { emission }_{\text {material }} \\
& + \text { ambient }_{\text {light model }} \times \text { ambient }_{\text {material }} \\
& +\sum_{i=0}^{n-1}\left(\frac{1}{k_{c} \pm k_{l} d \pm k_{q} d^{2}}\right)_{i} \times\left({\text { spotlighteffects })_{i}}\right. \\
& \times\left[\begin{array}{l}
\text { ambient }_{\text {light }} \times \text { ambient }_{\text {material }} \\
\pm(\max \{L \cdot n, 0\}) \times \text { diffuse }_{\text {light }} \times \text { diffuse }_{\text {material }} \\
+(\max \{s \cdot n, 0\})^{\text {shininess }} \times \text { specular }_{\text {light }} \times \text { specular }_{\text {material }}
\end{array}\right]_{i}
\end{aligned}
$$

\section{IMPLEMENTATION OF PARALLELIZED PHONG SHADING ALGORITHM}

Parallel algorithms vary in features according to different categories of problem and architectures of parallel machines. A well-worked parallel algorithm is adaptive to the characteristics of the structure of parallel computer hardware system and the inherent parallelism of the problems to solve. For parallel computing, there is a very important principle, that is to manage to increase the proportion of computing time and reduce the frequency of communication. If the communication of processing units can coincide, the execution efficiency of the program will be promoted greatly.

The parallelism of shading algorithm can be embodied in two aspects_-data parallelism and task parallelism[3],[4]. This paper puts forward a combined method with the strategy of both data and task parallelism, which is optimized in the pipelined design to achieve higher hardware utilization and decrease the intermediate data quantity. This parallel implementation is able to deal with data intensive tasks such as image processing and vertex shading, and make the system throughput speed up to a high level.

To accomplish the above calculation, the amount of computation is large. Traditional GPU using multiple vertex shading devices to do the calculation. The polymorphic array architecture processor (PAAG) [8] is used to achieve parallel shading in this paper .

\section{A. The Polymorphic Array Architecture Processor}

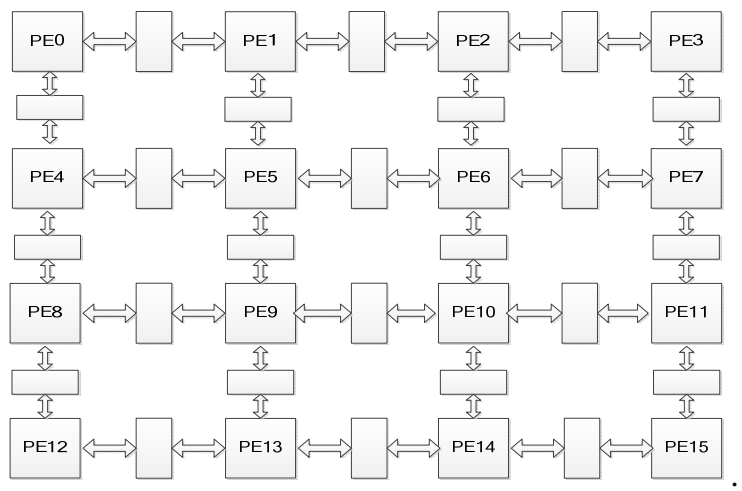

Figure 2. The structure of the polymorphic array architecture processor . 
As shown in Figure 2, it is an efficient parallel processing array and structure which is suitable for graphic image. The processing cell array structure is composed of multiple processor computer system clusters, each cluster is composed of 16 processing units with $4 * 4$ array. The processing unit is a two-dimensional array composed of the PE. Each line has line controllers, each column also has column controllers. This makes such a machine can achieve highly efficient parallel computing by line level, data-level parallel computing and parallel computing operating level.

In order to validate the program, we use PAAG processor simulation system, compilation system, debugging environment and the parameter extraction tool for performance analysis. This is a complete set of software development tools providing a reliable guarantee for program execution and performance analysis.

Clock accurate simulation techniques are used in PAAG system, the individual levels of PE have carried out modeling, pre-processing module distributed the stored data to the various functional modules according to the connection between the modules and the communication protocols, processed data is sent into the post-processing module, which delay the data according to the internal software counter clock appropriate and then send the data to a preprocessing module, forming a loop, to achieve the communication between the various modules and the analog clock, in order to achieve the clock accuracy level modeling. The compilation system provides a compiler system from high-level language to the compiler , and assembler instructions from assembly instructions to machine readable machine code assembler, Its debugging environment used to validate the correctness of the prototype system, and performance analysis tools achieve speedup with collecting the corresponding parameter extraction algorithm program in a single PE and the running time in several PEs, what's more, it provides the basis for the optimization algorithm.

\section{B. Parallel Algorithm Implementation}

1) Serial implementation: To get serial computation , vector standardization and the calculations of attenuation factor and spotlight effects should be achieved first.Then the calculations of diffuse light, specular light and ambient light component should be achieved. you can also change the order of them.the paper lists an order of the algorithm. Serial implementation is shown as figure3:

2) Parallelization preliminary: Parallel processing of lighting algorithms requires the work to be seperated into discrete sections, which helps to solve several program instructions timely,at any time or at the same time ,which will shorten the calculation time. Parallel algorithm devids the algorithm into multiple parts due to the formula in the adding portion ,ambient light, specular light, diffuse light and the attenuation factor would be calculated in every portion. Preliminary divided idea is shown as figure4:

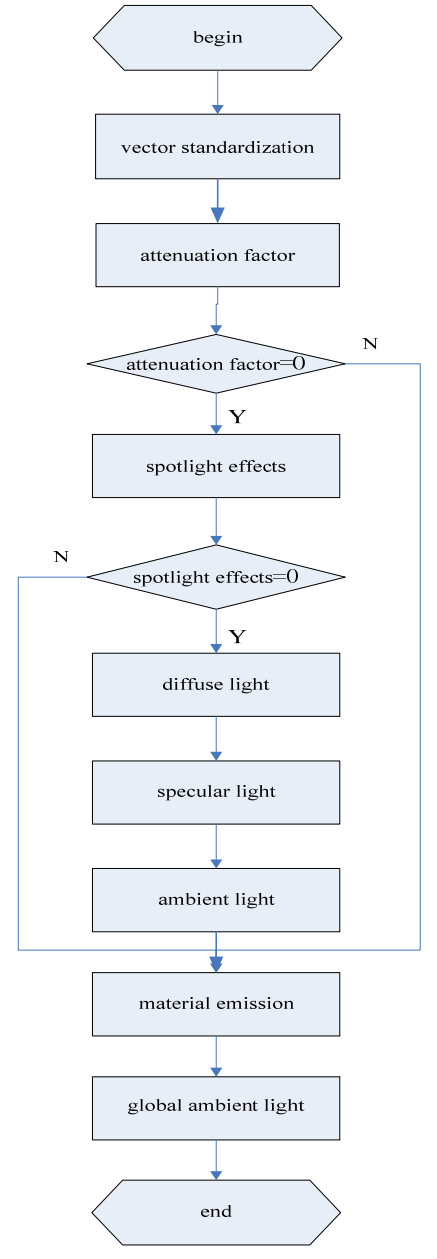

Figure 3. Serial structure .

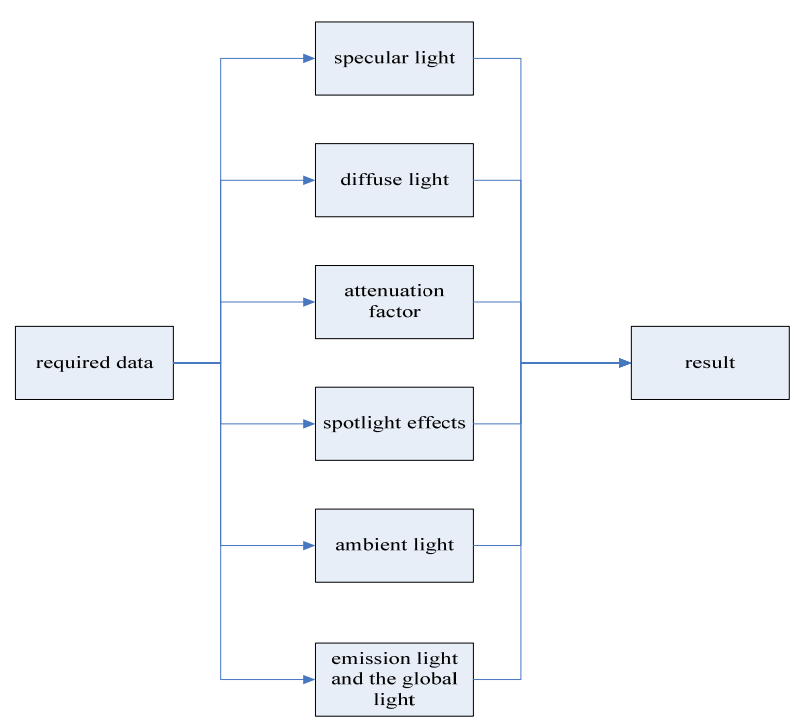

Figure 4. Preliminary parallelization structure. 
3) Parallelization implementation : Since the amount of the calculationg in each part could be different , the simple division will lead to load imbalance,which would affect efficiency of the algorithm, it uses a load more balanced parallel strategy, re-optimization tasks is shown in the following figure.The calculation of the vectors S1,S2(required by specular light) is put into another PE,the computing and the merging of emission light and the global light are put into the same PE, which ensurs the relevance of the data, Serial implementation is shown as follows :

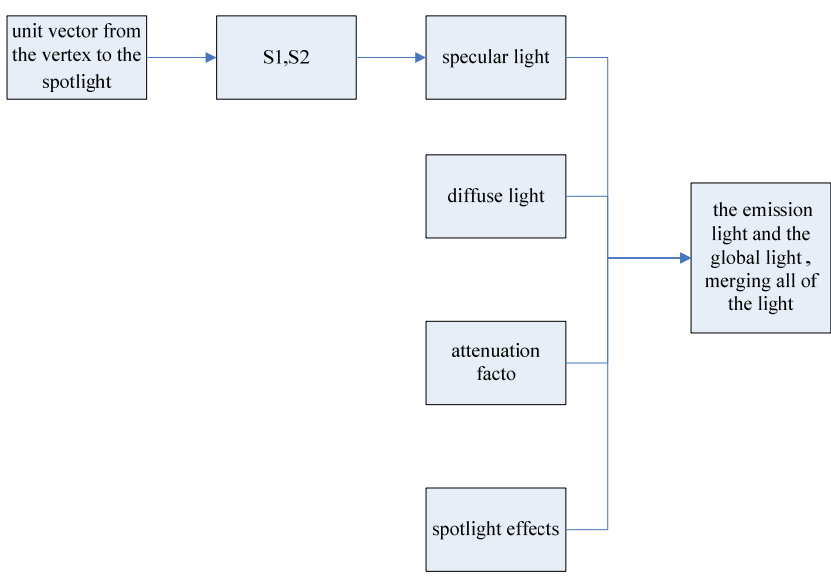

Figure 5. Parallelization structure

4) The implementation of parallelization algorithm: When we achieve the parallelization of The Phong shading algorithms, the source allocation and the full use of the source should be considered so that plura PE could be interdependent and synchronized. Specific implementation is shown below:

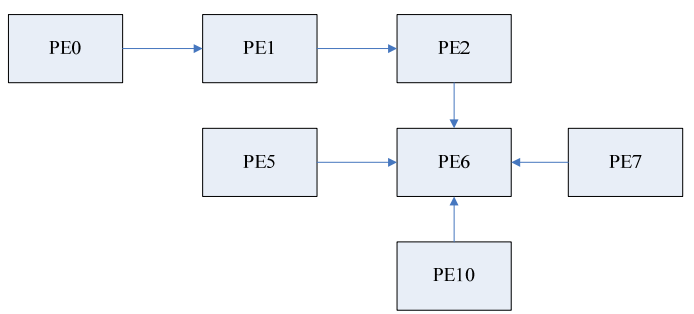

Figure 6. The structure of the communication between PE

The implementation of the parallelization used seven PE to complete:

a) PE0: Normalized the vector from the vertex to spotlight,then send results into PE1.

b) PE1: Calculate the sum of the distance between the vertex and the observation point and the distance between the vertex and the light source in the specuar light. First, determine whether there is specular light, after that, receive calculated the value of the desired unit vector from PE1 .Then, it is required to calculate again. What's more, the results should be normalized,calculate the required value of the specular light component, and finally send the result to PE2.

c) PE2: Do the specular light calculations. According to the calculation formula(3) of the specular light and the desired value obtained from PE1, calculated specular light ,send result to PE6.

d) PE5: Calculate the diffuse light. According to the formula(2) of the diffuse light, and the the results would be sent to PE6 from PE5.

e) PE7: Calculate the attenuation factor. According to the calculation formula(4) of the attenuation factor and the given values,we can get the attenuation factor.we should then determine whether the result is 0 . if it is, then only the material emitting light and the global ambient light could influence on the lighting effects, if it is not, The results will be sent into the PE6.

f) PE10: Calculate spotlight effects. First, determine whether the spotlight effect exists, if it exists, the spotlight effects are calculated according to the formula(6).Then we need to determine whether the result is 0 .If it is 0 , only the material emitting light and global ambient light influence on lighting effects, if it is not, results would be sent into PE6.

g) PE6: Calculate the ambient light and each of the above ingredients are combined,.Then add the material emitting light and global ambient light to get the final lighting color .

In summary, the final results are stored in PE6 and have achieved the lighting parallelization.

\section{RESUltS AND ANALYSIS}

With Amdahl's law [7], the system performance of improvement can be intuitively reflected by the value. It defines a speedup achieved by acceleration measures.

Speedup [6] reflects the rate between using accelerated measures to complete a task and not using accelerated measures to accomplish the same task to speed up. Therefore, the speedup can be defined as:

Speed $=\frac{\text { The performance of achieving the whole task when using accelerated measures }}{\text { The performance of achieving the whole task without using accelerated measures }}$

Can also be defined as :

Speed $=\frac{\text { The performance of achieving the whole task without using accelerated measures }}{\text { The performance of achieving the whole task when using accelerated measures }}$

It is convenient for us to estimate if the latter define is used.

Using different PE, amount and different assignments of different ways to get a speedup, the results would also be different. the following is a serial processing program of the vertex and results running parallel program 2,5,6,7 PEs: 
TABLE I. IMPLEMENTATION TIME WITH 1PE

\begin{tabular}{|c|c|c|c|}
\hline & $\begin{array}{c}\text { running } \\
\text { time(clock) }\end{array}$ & $\begin{array}{c}\text { block time } \\
\text { (clock) }\end{array}$ & $\begin{array}{c}\text { exec time } \\
\text { (clock) }\end{array}$ \\
\hline PE0 & 1368 & 0 & 1368 \\
\hline
\end{tabular}

TABLE II. IMPLEMENTATION TIME WITH 2PE

\begin{tabular}{|c|c|c|c|c|}
\hline & $\begin{array}{c}\text { running } \\
\text { time(clock) }\end{array}$ & $\begin{array}{c}\text { block time } \\
\text { (clock) }\end{array}$ & $\begin{array}{c}\text { exec time } \\
\text { (clock) }\end{array}$ & $\begin{array}{c}\text { speed } \\
\text { up }\end{array}$ \\
\hline PE0 & 444 & 0 & 444 & \multirow{2}{*}{1.45} \\
\cline { 1 - 4 } PE1 & 935 & 0 & 935 & \\
\hline
\end{tabular}

TABLE III. IMPLEMENTATION TIME WITH 5PE

\begin{tabular}{|c|c|c|c|c|}
\hline & $\begin{array}{c}\text { running } \\
\text { time(clock) }\end{array}$ & $\begin{array}{c}\text { block time } \\
\text { (clock) }\end{array}$ & $\begin{array}{c}\text { exec time } \\
\text { (clock) }\end{array}$ & $\begin{array}{c}\text { speedu } \\
\text { p }\end{array}$ \\
\hline PE0 & 391 & 0 & 391 & \multirow{2}{*}{1.77} \\
\cline { 1 - 4 } PE1 & 116 & 326 & 442 & \\
\cline { 1 - 4 } PE4 & 155 & 144 & 299 & \\
\cline { 1 - 4 } PE5 & 232 & 540 & 772 & \\
\hline
\end{tabular}

TABLE IV. IMPLEMENTATION TIME WITH 6PE

\begin{tabular}{|c|c|c|c|c|}
\hline & $\begin{array}{c}\text { running } \\
\text { time(clock) }\end{array}$ & $\begin{array}{l}\text { block time } \\
\text { (clock) }\end{array}$ & $\begin{array}{l}\text { exec time } \\
\text { (clock) }\end{array}$ & $\begin{array}{l}\text { speed } \\
\text { up }\end{array}$ \\
\hline PEO & 391 & 0 & 391 & \multirow{6}{*}{2.65} \\
\hline PE1 & 117 & 326 & 443 & \\
\hline PE4 & 144 & 155 & 299 & \\
\hline PE5 & 239 & 277 & 516 & \\
\hline PE6 & 307 & 0 & 307 & \\
\hline PE9 & 382 & 0 & 382 & \\
\hline
\end{tabular}

TABLE V. IMPLEMENTATION TIME WITH 7PE

\begin{tabular}{|c|c|c|c|c|}
\hline & $\begin{array}{c}\text { running } \\
\text { time(clock) }\end{array}$ & $\begin{array}{l}\text { block time } \\
\text { (clock) }\end{array}$ & $\begin{array}{l}\text { exec time } \\
\text { (clock) }\end{array}$ & $\begin{array}{c}\text { speedu } \\
\text { p }\end{array}$ \\
\hline PEO & 199 & 0 & 199 & \multirow{7}{*}{2.87} \\
\hline PE1 & 204 & 148 & 352 & \\
\hline PE2 & 117 & 287 & 404 & \\
\hline PE5 & 323 & 0 & 323 & \\
\hline PE6 & 238 & 239 & 477 & \\
\hline PE7 & 307 & 0 & 307 & \\
\hline PE10 & 383 & 0 & 382 & \\
\hline
\end{tabular}

The changement of speedup is shown as follows:

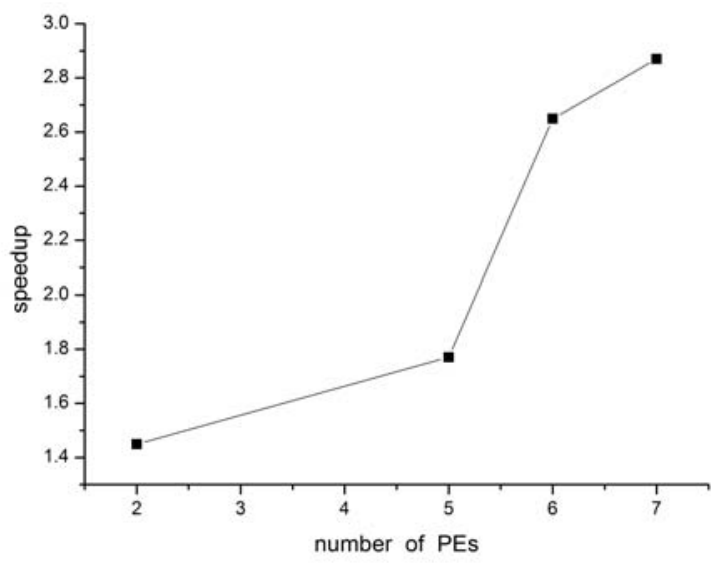

Figure 7. The changement of speedup

From the figure above, we can see that when the PE is 7, the speedup is relatively high.

\section{SUMMARY}

This study and the experimental results show that the parallelization of lighting can better make use of resources, i.e.the processing power of multicore processors. The serial computation is shifted into optimization problems of parallel computing program. The result accelerats the original operation, as well as improve operational efficiency. In further studies, the algorithm can be optimized better to enhance the efficiency of the algorithm. Also we can better streamline the functional modules, so that the vertex shading processors are arranged more compact, which can be implemented multi-sets of vertices shading in a cluster to improve resource utilization.

\section{ACKNOWLEDGEMENT}

This research is supported by a key grant from the China Natural Science Foundation (No. 61136002)

\section{REFERENCES}

[1] Dave Shreiner , The Khronos OpenGL ARB Working Group.OpenGL Programming Guide.China Machine Press, 2010.

[2] Edward Angel.Interactive Computer Graphics.Tsinghua University Press, 2006.

[3] Peter S.Pacheco. An Introduction to Parallel Programming .China Machine Press, 2011.

[4] Bo Dai.The Research of Parallel Algorithm And Applicationg[ D] / /Chengdo: University of Electronic Science And Technology of China, 2002.

[5] Zhen Yao. Study on Parallel Programming Models[ D] / / Hefei: University of Science and Technology of China.

[6] Chao Xie,Lian Dao Mai.Research and Analysis of Parallel Computing System Speedup[J].Computer Engineering and Applications,2003 : 66-68.

[7] Amdahl G. Validity of the single-processor approach to achieving large scale computing capailities. In; Ptoc AFIPS Conf. Atlantic City. $1967.483-485$. 
[8] Hucai Huang, Tao Li, Jungang. Han, "Simulator Implementation and Performance Study of A Polymorphous Array Computer", 2013 12th
IEEE International Conference on Trust, Security and Privacy in Computing and Communications, Melbourne,Australia, pp1848-1855. 